\title{
Cold denaturation of a protein dimer monitored at atomic resolution
}

\author{
Mariusz Jaremko 1,2,6, Łukasz Jaremko,3,6, Hai-Young Kim', Min-Kyu Cho', Charles D Schwieters ${ }^{4}$, \\ Karin Giller', Stefan Becker' \& Markus Zweckstetter1,5*
}

\begin{abstract}
Protein folding and unfolding are crucial for a range of biological phenomena and human diseases. Defining the structural properties of the involved transient species is therefore of prime interest. Using a combination of cold denaturation with NMR spectroscopy, we reveal detailed insight into the unfolding of the homodimeric repressor protein CyIR2. Seven threedimensional structures of CyIR2 at temperatures from $25^{\circ} \mathrm{C}$ to $-16{ }^{\circ} \mathrm{C}$ reveal a progressive dissociation of the dimeric protein into a native-like monomeric intermediate followed by transition into a highly dynamic, partially folded state. The core of the partially folded state seems critical for biological function and misfolding.
\end{abstract}

Dandir artially folded species have a key role in the folding, unfolding and misfolding of proteins ${ }^{1,2}$. Even for small proteins, protein folding intermediates can slow the rate of folding and lead to misfolding of proteins implicated in neurodegenerative disorders ${ }^{1,3}$. Increasing evidence suggests that pathogenic misfolding of globular proteins occurs from partially unfolded states, which must be populated to permit oligomerization and amyloid fibril formation ${ }^{4-6}$. Owing to their transient nature, the structural characterization of protein folding intermediates is a challenge ${ }^{1,2}$. Protein folding intermediates can be detected by analysis of the influence of denaturants on the folding and unfolding kinetics ${ }^{7}$. Native state hydrogen exchange has been extremely powerful in revealing protein folding intermediates ${ }^{3}$. Moreover, native-state alkyl-proton exchange allows mapping of side chain exposure in partially unfolded forms $s^{8}$. Transition states in protein folding can be probed via the effect of point mutations on the folding kinetics, and very slow folding events may be monitored directly in real time ${ }^{10}$. Careful changes in solution conditions and site-specific mutations can be used to trap transient folding intermediates by specifically destabilizing the native state. Indeed, protein engineering directed by hydrogen exchange measurements has allowed the determination of threedimensional structures of folding intermediates of redesigned proteins including apo cytochrome $b_{562}$, engrailed homo domain, T4 lysozyme and RNase H using NMR spectroscopy ${ }^{11-15}$. Moreover, protein engineering in combination with rapid mixing and stoppedflow experiments suggested that the presence of protein folding intermediates is connected to the simultaneous requirement for function and folding efficiency ${ }^{16}$. The three-dimensional structures of the intermediates determined thus far, together with a large body of equilibrium and kinetics data, have provided unique insights into the mechanisms of protein folding ${ }^{1,2}$. However, in many cases the intermediates show substantial exchange broadening of NMR signals that arises from fluctuations between different conformations, complicating their structural characterization ${ }^{17,18}$.

A powerful approach for the detection and structural characterization of rare and transiently populated non-native states is NMR spin relaxation analysis ${ }^{19-21}$. NMR relaxation dispersion allows insights into conformational exchange reactions and provides access to chemical shifts and bond vector orientations of excited states $^{22}$. Because of the unique sensitivity of NMR relaxation dispersion, backbone folds of transiently formed intermediates, which comprise only $0.5 \%$ of the total population of molecules, can be determined ${ }^{6,23,24}$. Moreover, large parts of folding landscapes can be probed when NMR relaxation dispersion measurements are combined with site-directed mutagenesis ${ }^{25}$. The studies have underscored that the function of proteins critically depends on their ability to populate different molecular conformations ${ }^{26}$.

Further insight into protein folding intermediates can be obtained by all-atom molecular dynamics simulations that can fold small fastfolding proteins ${ }^{27}$. However, the structural properties of the unfolded state and the intermediates that are observed during the folding simulation can be strongly influenced by the choice of force field ${ }^{28}$. Thus, the combination of molecular dynamics simulations with a wide variety of experimental restraints has become highly useful ${ }^{16}$.

Both high and low temperatures can lead to unfolding of proteins. Heat denaturation generally occurs by a two-state process excluding direct access to intermediate states. In contrast, decreasing temperatures can induce unfolding of proteins in a noncooperative fashion ${ }^{29-31}$, and intermediate states can be sufficiently populated to allow their structural characterization. Thus, unique structural information about intermediate states might be obtained at low temperatures. At the same time, cold denaturation occurs for most monomeric proteins below $-20{ }^{\circ} \mathrm{C}$, such that additional destabilization of the protein by mutation, high pressure, nonphysiological solvents or encapsulation in reverse micelles is required to observe unfolding $29,30,32,33$. For example, site-directed mutagenesis allowed the NMR-based characterization of cold-unfolded states of the protein barstar ${ }^{33}$. Cold-unfolded states of ubiquitin were observed when the protein was encapsulated in reverse micelles ${ }^{29}$, even though the protein is highly stable toward low temperature in buffer $^{34}$. Moreover, molten globule-like states of some proteins are prone to cold denaturation ${ }^{35}$.

Here we show that the combination of cold-induced unfolding and NMR spectroscopy allows visualization of the unfolding intermediates of the homodimeric protein CylR2 at atomic resolution. CylR2 is a key regulator of the production of the exotoxin

'Department for NMR-based Structural Biology, Max Planck Institute for Biophysical Chemistry, Göttingen, Germany. ${ }^{2}$ Institute of Biochemistry and Biophysics, Polish Academy of Sciences, Warsaw, Poland. ${ }^{3}$ Faculty of Chemistry, Warsaw University, Warsaw, Poland. ${ }^{4}$ Division of Computational Bioscience, Center for Information Technology, US National Institutes of Health, Bethesda, Maryland, USA. ${ }^{5}$ German Center for Neurodegenerative Diseases (DZNE), Göttingen, Germany. ${ }^{6}$ These authors contributed equally to this work. *e-mail: Markus.Zweckstetter@dzne.de 
a
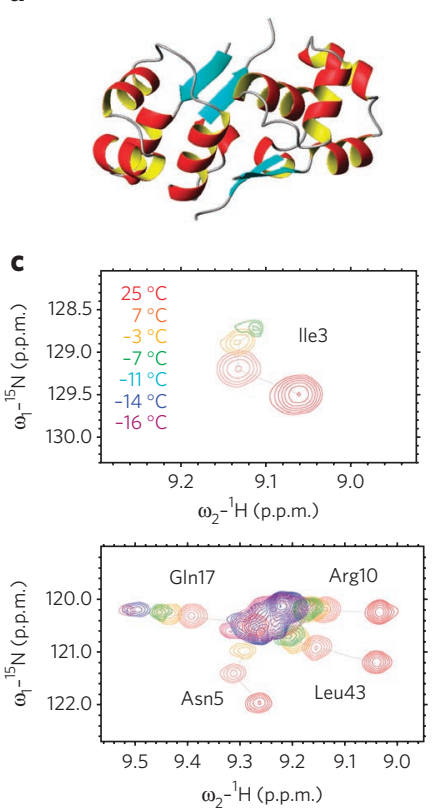
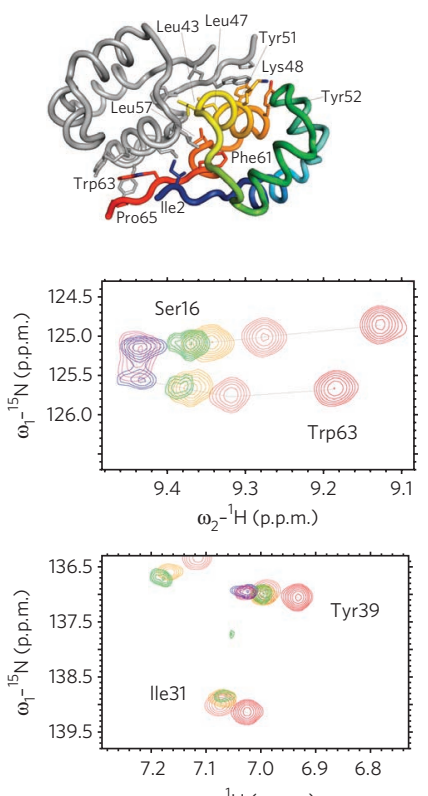

$\omega_{2}-{ }^{1} \mathrm{H}$ (p.p.m.) b

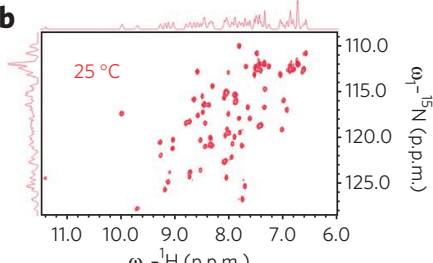

$\omega_{2}-{ }^{1} \mathrm{H}$ (p.p.m.)

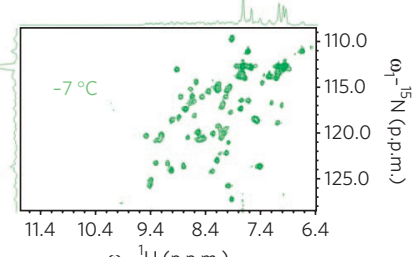

$\omega_{2}-1$ H (p.p.m.)
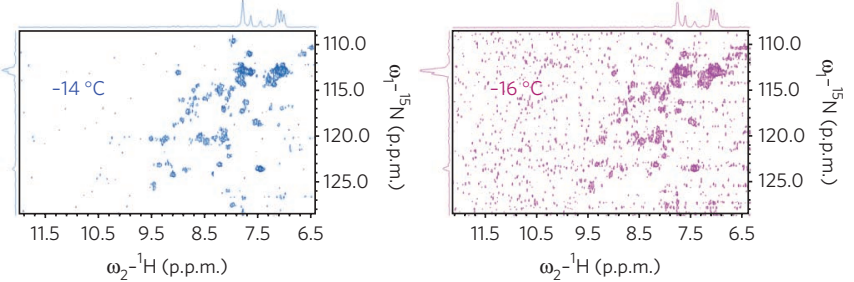

Figure 1 | Temperature sensitivity of the CyIR2 homodimer. (a) Three-dimensional structure of the native state of CyIR2; ribbon representation (left); key side chains buried in the dimer interface are highlighted (right). (b) Two-dimensional ${ }^{1} \mathrm{H}-{ }^{15} \mathrm{~N} \mathrm{HSQC}$ spectra of CyIR2 at decreasing temperatures. (c) Superposition of selected regions from $\mathbf{b}$.

cytolysin in Enterococcus faecalis, an opportunistic bacterium that has become one of the most troublesome hospital pathogens ${ }^{36}$. On the basis of a large number of inter-residue contacts, we determined the three-dimensional structures of six intermediate states that were populated during cold-induced unfolding of CylR2. The structures reveal a progressive dissociation of the native-state dimer (Fig. 1a) into a monomeric intermediate with native-like topology and nonnative side chain contacts. At the lowest temperature, CylR2 assumes a monomeric, partially folded state that seems critical for biological function and misfolding.

\section{RESULTS}

\section{Cold-induced transformation into a dynamic ensemble}

CylR2 is a stable homodimeric protein with a heat denaturation midpoint of $77.5{ }^{\circ} \mathrm{C}$ (Supplementary Results, Supplementary Fig. 1a). At low temperatures approaching $-8{ }^{\circ} \mathrm{C}$, only very small changes were observed in the CD signal (Supplementary Fig. 1b). The predominantly helical structure of CylR2 was therefore retained at low temperatures.

Next, we probed the impact of temperature on the structure of CylR2 using NMR spectroscopy. To reach temperatures as low as $-16{ }^{\circ} \mathrm{C}$ without freezing, glass capillaries with a $1.0-\mathrm{mm}$ outer diameter were used ${ }^{37}$. Two-dimensional ${ }^{1} \mathrm{H}-{ }^{15} \mathrm{~N}$ HSQC spectra were recorded for several different temperatures in the range of $25^{\circ} \mathrm{C}$ to $-16^{\circ} \mathrm{C}$ (Fig. 1b,c). Figure $\mathbf{1 b}$ shows that the quality of the spectra decreased with decreasing temperature because of severe line broadening. In particular, at $-16^{\circ} \mathrm{C}$, most backbone amide proton-amide cross-peaks were only slightly above the noise level, and for some residues the resonances were lost (Supplementary Fig. 2). Severe broadening of NMR lines is often observed in protein folding intermediates owing to interconversion between different conformations at an exchange rate that is intermediate on the NMR timescale ${ }^{17}$. In addition, ${ }^{1} \mathrm{H}_{-}{ }^{15} \mathrm{~N}$ chemical shifts changed nonlinearly for several residues such as Ile3, Asn5 and Trp63 (Fig. 1c), a further sign of conformational exchange. Notably, NMR spectra obtained upon cooling to $-16^{\circ} \mathrm{C}$ and reheating to $25^{\circ} \mathrm{C}$ were in agreement with spectra originally obtained at $25^{\circ} \mathrm{C}$, showing complete reversibility over the course of the experiments (data not shown).

To obtain insight into changes in protein dynamics, we performed NMR relaxation measurements. The steady-state ${ }^{15} \mathrm{~N}-{ }^{1} \mathrm{H}$ NOE is highly sensitive to motions occurring on ps-to-ns timescales. In line with the rigid nature of the CylR2 dimer, the average steadystate ${ }^{15} \mathrm{~N}-{ }^{1} \mathrm{H}$ NOE was $0.81 \pm 0.05$ at $25{ }^{\circ} \mathrm{C}$ (Fig. 2a). Upon lowering the temperature to $-3{ }^{\circ} \mathrm{C},{ }^{15} \mathrm{~N}-{ }^{1} \mathrm{H}$ NOE values of most residues experienced only small changes, with the average value remaining at $0.81 \pm 0.13$ (Fig. 2b). Exceptions were Glu66 and Thr55, whose ${ }^{15} \mathrm{~N}-{ }^{1} \mathrm{H}$ NOEs decreased by more than 0.4 . The strongly reduced ${ }^{15} \mathrm{~N}-{ }^{1} \mathrm{H}$ NOE values of these two residues point to a slight perturbation of the CylR2 structure at $-3{ }^{\circ} \mathrm{C}$. A further reduction of the temperature to $-11^{\circ} \mathrm{C}$, however, induced substantial changes across the complete CylR2 sequence (Fig. 2c). For all residues, ${ }^{15} \mathrm{~N}-{ }^{1} \mathrm{H}$ NOE values were lower, resulting in an average value of $0.27 \pm 0.07$ (Fig. 2c and Supplementary Table 1). The measurements demonstrate that ps-to-ns dynamics of the CylR2 backbone were strongly increased at $-11{ }^{\circ} \mathrm{C}$. In addition, the observed line broadening (Fig. $\mathbf{1 b}$ and Supplementary Fig. 3) indicated that extensive dynamics were also present on longer timescales, which preclude a quantitative characterization of the exchange processes. We conclude that CylR2 populates a highly dynamic ensemble of conformers at $-11^{\circ} \mathrm{C}$.

\section{From dimer to monomer}

Next, we probed the oligomerization state of CylR2 using diffusion NMR spectroscopy. In agreement with the three-dimensional structure of CylR2, we obtained a hydrodynamic radius of $\sim 22.6 \AA$ at $25^{\circ} \mathrm{C}$ and above (Fig. 3a). Below $-3{ }^{\circ} \mathrm{C}$, the ensemble-averaged value of the hydrodynamic radius progressively decreased and reached a value of $16.2 \AA$ at $-10^{\circ} \mathrm{C}$. On the basis of the experimental values, we calculated the molar ratio of the dimer and monomer concentration for each temperature using the relation between the hydrodynamic radius and the concentration of the monomeric and dimeric species (further details are in Online Methods). The quantitative analysis revealed that $92-94 \%, 42-51 \%$ and $0.8-1.4 \%$ of CylR 2 molecules were present as dimers at $-3{ }^{\circ} \mathrm{C},-7^{\circ} \mathrm{C}$ and $-11^{\circ} \mathrm{C}$, respectively. 
a

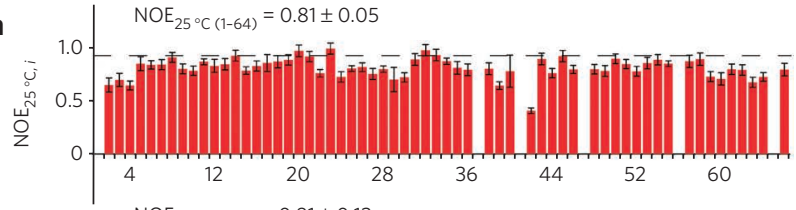

b

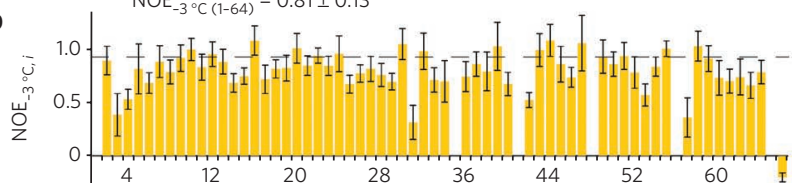

C

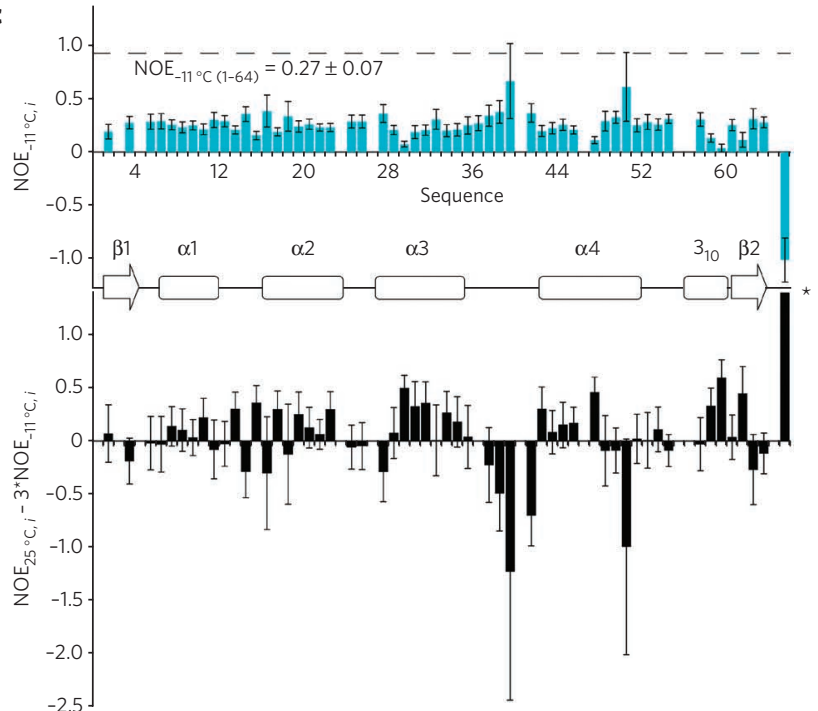

Figure 2 | Dynamics of protein folding intermediates of CyIR2.

(a,b) Steady-state ${ }^{15} \mathrm{~N}-{ }^{1} \mathrm{H} N O E$ at $25^{\circ} \mathrm{C}(\mathbf{a})$ and $-3{ }^{\circ} \mathrm{C}(\mathbf{b})$. (c) Steadystate ${ }^{15} \mathrm{~N}-1 \mathrm{H}$ NOE at $-11^{\circ} \mathrm{C}$ and the normalized difference between the two states $\left(\mathrm{NOE}_{25}{ }^{\circ} \mathrm{C}, i-3 \cdot \mathrm{NOE}_{-11^{\circ} \mathrm{C}, i}\right)$. The secondary structure of the native state is indicated. The value of Glu66 is outside of the scale (marked with an asterisk). Error bars for the ith ${ }^{15} \mathrm{~N}-{ }^{-1} \mathrm{H}$ correlation at temperature $T$ were derived from its $S / N$ value and peak height values with $\left(I_{\text {NOE }}\right)$ and without $\left(I_{\text {nONOEE }}\right){ }^{1} \mathrm{H}$ presaturation according to the equation $\Delta_{\text {NOE, T,i }}=$ $\left|N O E_{i}\right| \cdot\left[1 /\left(S / N_{\text {NOEi }}\right)^{2}+1 /\left(S / N_{\text {nONOE }}\right)^{2}\right]^{1 / 2}$, where $N^{\prime} E_{i}=I_{\text {NOEE }} / I_{\text {nONOEi }}$. In the case of the normalized difference in $\mathbf{c}$, error bars were determined as $\Delta_{\text {NOEi }}=$ $\left[\left(\Delta_{\mathrm{NOE}, 25^{\circ}, i}\right)^{2}+\left(3 \cdot \Delta_{\mathrm{NOE},-11^{\circ}, \mathrm{i},}\right)^{2}\right]^{0.5}$.

Changes in the global correlation time further supported the cold-induced dissociation of the CylR2 dimer (Fig. 3b and Supplementary Fig. 4). At $25^{\circ} \mathrm{C}$, we observed a global correlation time of $8.7 \pm 0.9 \mathrm{~ns}$, in line with an expected value of $\sim 9 \mathrm{~ns}$ for a 15.4-kDa protein (http://www.nmr2.buffalo.edu/). Owing to the increase in solution viscosity with decreasing temperature, the global correlation time increased when the temperature was lowered to $-3{ }^{\circ} \mathrm{C}$. Below $-3{ }^{\circ} \mathrm{C}$, however, the global correlation time started to drop and reached a value of approximately $15 \mathrm{~ns}$ at $-15^{\circ} \mathrm{C}$. Together the data demonstrate that CylR2 populates a monomeric, highly dynamic state in supercooled solution.

To reveal structural changes upon cooling at atomic resolution, we solved the three-dimensional structures of CylR2 at six different temperatures down to $-16{ }^{\circ} \mathrm{C}$ using NOE-based NMR procedures (Figs. 3c and 4a, Supplementary Fig. 5, Supplementary Table 2 and Supplementary Video 1). At $25^{\circ} \mathrm{C}$, a large number of restraints resulted in a highly defined structure of the native CylR2 dimer (Supplementary Table 3 and Fig. 4b). Upon cooling from $25^{\circ} \mathrm{C}$ to $-3^{\circ} \mathrm{C}$, most NOE contacts were unchanged. However, the mobility of the $\mathrm{C}$ terminus increased, and NOE contacts between the side chain of Pro65 in one subunit of the CylR2 dimer and the aromatic
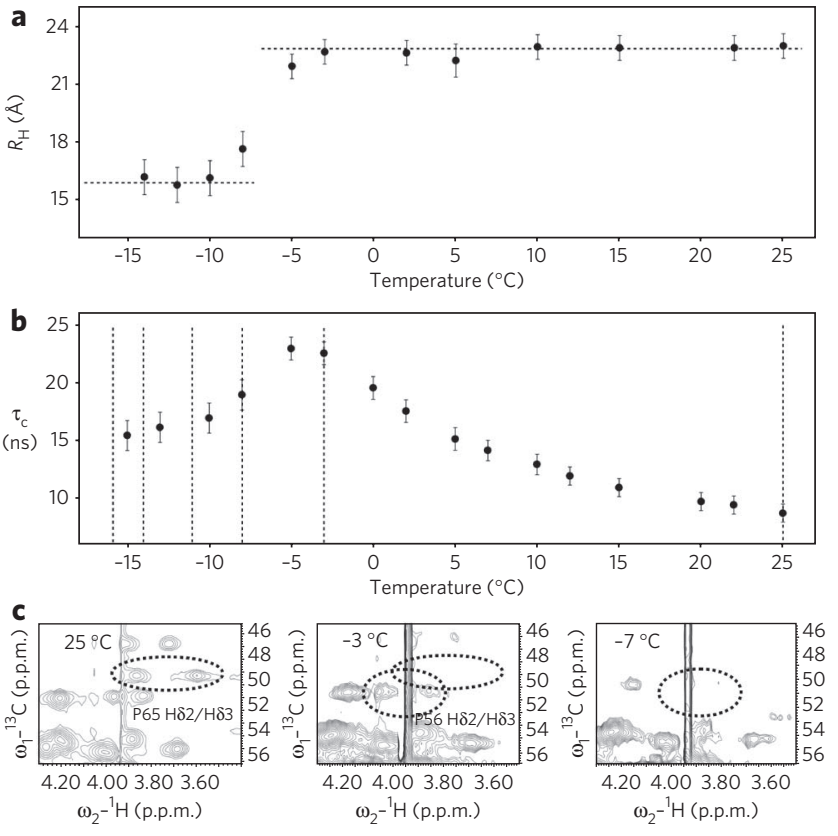

Figure 3 | Cold-induced dissociation of the native dimer.

(a) Hydrodynamic radius $\left(R_{H}\right)$ as a function of temperature. Average values (dashed lines) for the CyIR2 dimer and monomer are $22.6 \AA$ and $16.1 \AA$, respectively. (b) Global correlation time as a function of temperature. Vertical lines indicate the temperatures at which threedimenional structures were solved. (c) Expanded small region of ${ }^{13} \mathrm{C}-{ }^{1} \mathrm{H}$ HSQCs at three temperatures. Disappearing cross-peaks are highlighted.

ring of Tyr51 in the other subunit were lost, suggesting an increase in distance between the two side chains and the sampling of more heterogeneous conformations (Figs. $3 \mathbf{c}$ and $\mathbf{4 b}$ ). The observed decrease in the number of intermolecular contacts (Supplementary Table 3) reveals a destabilization of the dimer interface at the onset of unfolding of CylR2.

At $-7{ }^{\circ} \mathrm{C}$, approximately $42-51 \%$ of CylR 2 molecules remain in a dimer state (Fig. 3a,b), in agreement with the continuous presence of the intermolecular $\mathrm{C}-\mathrm{H}-\pi$ interaction between Trp63 from one subunit and Lys48 from the second subunit (Supplementary Fig. 6). Comparison with the NOE spectra of the native state unambiguously identified about $50 \%$ of the intermolecular contacts of the native state that can exclusively be attributed to dimeric CylR2 (Supplementary Table 3). In contrast, intramolecular NOE contacts have contributions from both monomeric and dimeric CylR2, as the two states were in fast exchange (Fig. 1b,c). Thus we performed ensemble calculations in which the experimental NOE restraints have to be fulfilled by an ensemble comprising a CylR2 monomer and a CylR2 dimer, that is, they must be fulfilled simultaneously by two structures (Fig. 4 and Supplementary Table 3). The resulting monomeric structure and dimeric subunit were similar: the r.m.s. deviation between their lowest energy structures was $0.64 \AA$ for the backbone atoms of residues 6-60. Notably, non-native NOE contacts present at $-11{ }^{\circ} \mathrm{C}$ (Fig. 5a) (described below) were not detected at $-7^{\circ} \mathrm{C}$ (Fig. 5b), indicating that non-native contacts were not sufficiently populated at $-7^{\circ} \mathrm{C}$ and confirming the high similarity of the monomer and dimer, which equally coexist in the fast exchange regime at $-7^{\circ} \mathrm{C}$. The CylR2 dimer at $-7^{\circ} \mathrm{C}$ differs from the native-state dimer by increased distances between the $\mathrm{C}_{\alpha}$ atoms of the two Leu57 residues and between the side chain of Pro65 in one subunit and the aromatic ring of Tyr51 in the other subunit (Supplementary Table 4). In addition, the four hydrogen bonds of Ile3, Asn5, Gln62 and Gln64, which stabilize the intramolecular 
a

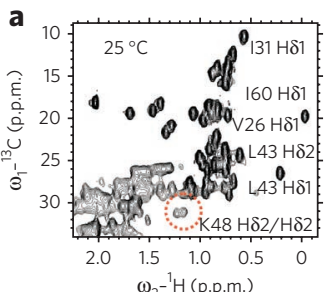

b
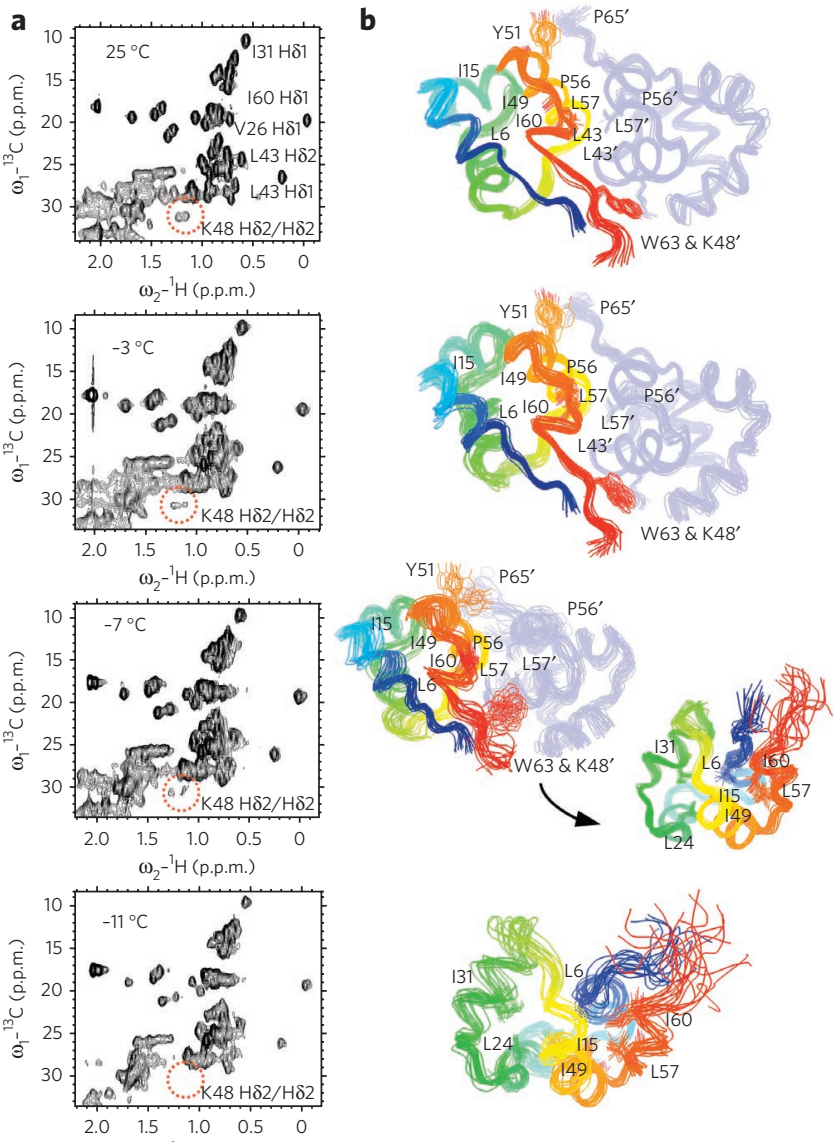

$\omega_{2}-{ }^{1} \mathrm{H}$ (p.p.m.)
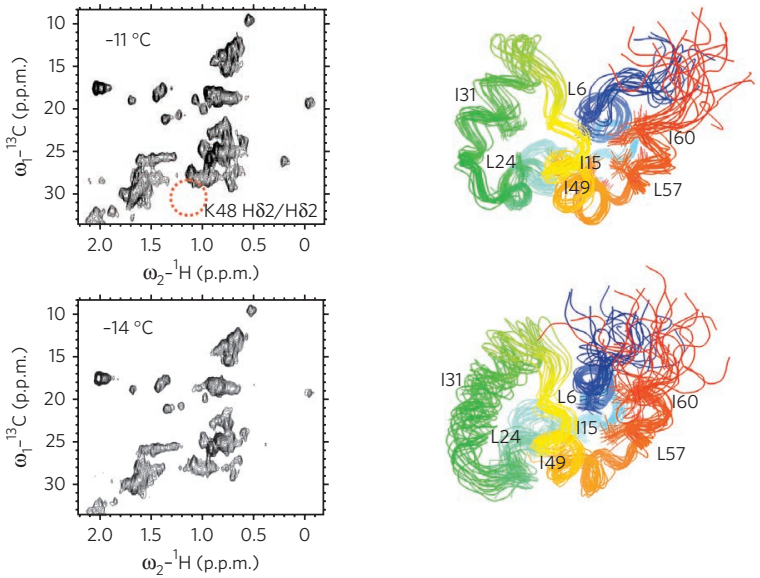

intermolecular C-H- $\pi$ interaction between Trp63 and Lys48 vanished (Fig. 4a). The three-dimensional structure of the monomeric intermediate of CylR2 was accurately defined by 360 intramolecular medium and long-range NOE distance restraints (Fig. $\mathbf{4 b}$ and Supplementary Table 3). The fold of the monomeric intermediate was similar to the structure of a CylR2 subunit in the native state and the CylR2 monomer at $-7^{\circ} \mathrm{C}$ (Supplementary Table 3). However, backbone dynamics were strongly increased (Fig. 2c), and the native-state intramolecular $\beta$-sheet was lost.

Several observations support the site-specific changes observed in the structures of the native-like monomeric state: (i) contacts between side chain atoms were observed in regions where backbone resonances were broadened (Supplementary Fig. 8); (ii) intersubunit NOE contacts disappear faster than long- and medium-range contacts within a subunit (Supplementary Fig. 9); (iii) the loss of intersubunit NOE contacts is consistent with changes in global correlation time and hydrodynamic radius (Fig. 3a,b); (iv) selected resonances, such as those belonging to the $\beta$-strand, disappear (Figs. 1d, 3c and 4a); and (v) unique non-native contacts were detected (described below). To further validate the threedimensional structure of the native-like monomeric intermediate, we used paramagnetic relaxation enhancement (PRE) $)^{38}$. MTSL (1-oxy-2,2,5,5-tetramethyl-D-pyrroline-3-methyl)-methanethiosulfonate) was attached to the single-cysteine mutants carrying mutations T55C and N40C. Overall, the experimental PRE profiles at $25{ }^{\circ} \mathrm{C}$ and $-10{ }^{\circ} \mathrm{C}$ were comparable (Supplementary Fig. 10). However, the residue range next to the MTSL-tagged residue that was already broadened beyond detection at $25^{\circ} \mathrm{C}$ was increased in the monomeric intermediate. This suggests that the MTSL tag samples a wider conformational space, in agreement with a more dynamic structure of the CylR2 intermediate. None of the structures from the bundle of conformers at $25{ }^{\circ} \mathrm{C},-3{ }^{\circ} \mathrm{C}$ and $-11{ }^{\circ} \mathrm{C}$ (Fig. 4) showed any violations of the PRE-derived distance restraints. In addition, the experimental PRE profiles agreed with profiles predicted from the NOE-based ensembles of structures (Supplementary Fig. 10). Slight deviations from the experimental profiles were due to approximation of the position of the paramagnetic center of MTSL for PRE prediction (Supplementary Fig. 10). Residual dipolar couplings in the backbone of CylR2 could not be obtained below $-7^{\circ} \mathrm{C}$ because of severe line broadening (Fig. 1b).

Non-native contacts in intermediate species are of prime importance for protein folding and misfolding ${ }^{1,6,11,12,14,16,23,24}$. In the case of CylR2, support for the presence of non-native contacts in the monomeric intermediate comes from ps-to-ns dynamics of the backbone (Fig. 2c). For residues $38-42$, steady-state ${ }^{15} \mathrm{~N}-{ }^{-1} \mathrm{H}$ NOEs at $-11^{\circ} \mathrm{C}$ were larger than in surrounding regions, in contrast to the dynamic properties in the native state. Detailed analysis of the NOE spectra revealed the basis for this increased rigidity. In the native state, Leu43 and Leu57 were involved in key intermolecular interactions stabilizing the dimer interface (Fig. 1a). Upon complete dimer dissociation, the side chains of Leu43 and Leu57 flip into the hydrophobic environment of the monomeric core and form non-native intramolecular contacts with Ile60 and Leu6, respectively (Fig. 5a and Supplementary Fig. 11). The non-native contacts were accompanied by a rotation of the side chain of Phe61 toward the solvent. Pro41 inserts into the open space left by the side chain of Phe61, resulting in a slight increase in the rigidity of residues 38-42 compared to neighboring regions.

\section{D structure of the monomeric folding intermediate}

At even lower temperatures, the protein monomer transforms into an ensemble of conformations that populates partial native-like but highly dynamic secondary structures (Fig. 6). Despite the disturbance of native-like packing of side chains, 99 structure-dependent contacts were identified at $-16{ }^{\circ} \mathrm{C}$-the lowest possible temperature with analyzable NOE spectra-corresponding to $12 \%$ of the
The native-like monomeric state

At $-11{ }^{\circ} \mathrm{C}$, less than $2 \%$ of dimeric CylR2 molecules remained (Fig. 3a,b), no intermolecular contacts were detected and the 
a

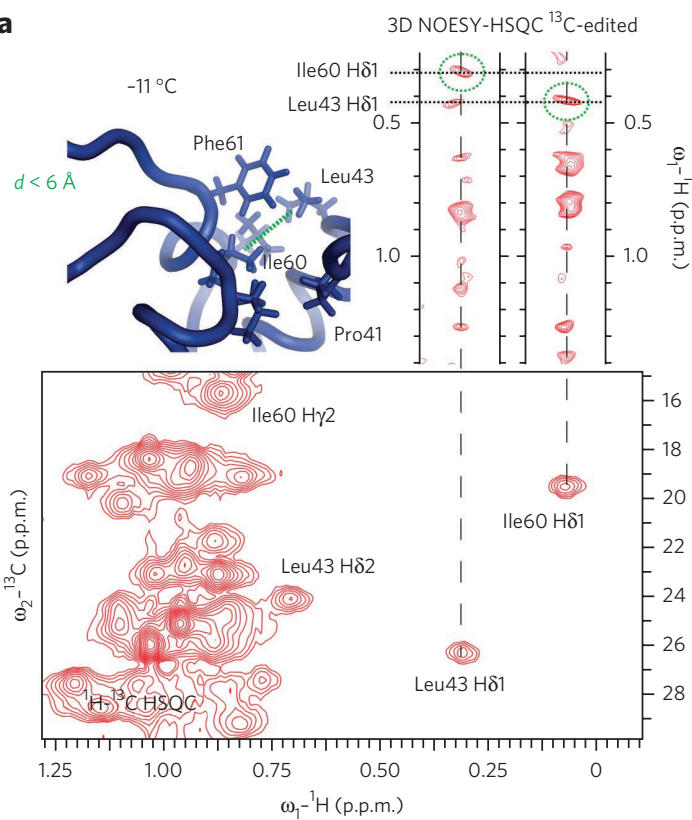

b

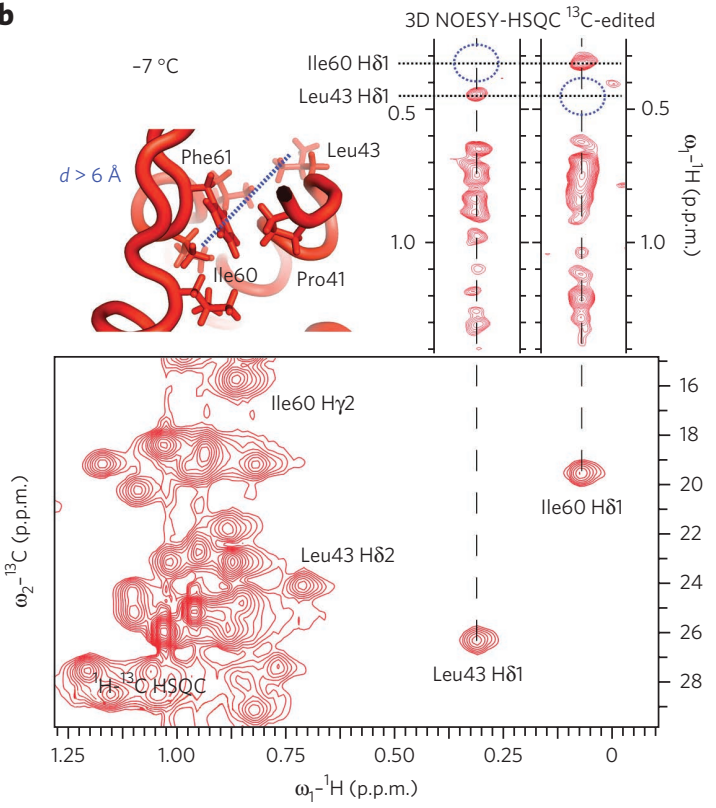

Figure 5 | Non-native contacts in the monomeric protein folding intermediate. (a) Non-native NOE contacts between well-separated Leu43 and 11 e60 at $-11^{\circ} \mathrm{C}$. (b) Corresponding strips from the three-dimensional (3D) ${ }^{13} \mathrm{C}$-edited NOESY-HSQC spectrum recorded at $-7{ }^{\circ} \mathrm{C}$ demonstrating the absence of the non-native contacts. The two-dimensional ${ }^{1} \mathrm{H}-{ }^{13} \mathrm{C}$ HSQC spectra show that the $\mathrm{H} \delta 1$ protons of Leu 43 and lle60 are well separated at both temperatures.

long-range contacts in the native state (Supplementary Table 3 and Supplementary Fig. 9). The core of the partially folded state is formed by helices $\alpha 1, \alpha 4$ and $3_{10}$ (Figs. 4 and 6a,b), which are stabilized by medium- and long-range contacts between Leu6, Ile15, Ile49 and Ile60. Mutation of either Leu6 or Ile49 to serine induced strong changes in the chemical environment across the protein at $25^{\circ} \mathrm{C}$, supporting their involvement in the hydrophobic core of the native state (Supplementary Fig. 12). Despite its lower definition, the helix-turn-helix motif was still observed at $-16^{\circ} \mathrm{C}$, in agreement with the presence of helix-like carbon chemical shifts (Supplementary Figs. 13 and 14). However, long-range contacts between the helix-turn-helix motif and the core formed by helices
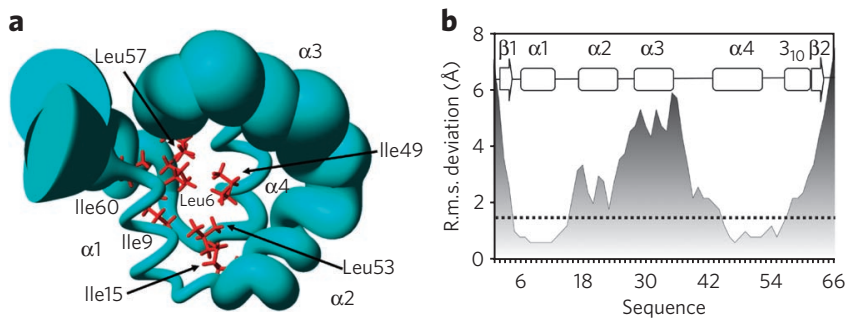

Figure 6 | Three-dimensional structure of a highly dynamic, partially folded state. (a) Sausage model of the dynamic, partially folded monomer at $-16^{\circ} \mathrm{C}$. Side chains that are involved in long- and medium-range contacts are shown as sticks. (b) R.m.s. deviation of $\mathrm{N}, \mathrm{CA}$ and $\mathrm{CO}$ atoms within the bundle of the 20-lowest energy conformers at $-16^{\circ} \mathrm{C}$. The secondary structure of the native state is indicated.

$\alpha 1, \alpha 4$ and $3_{10}$ were lost. The loss of long-range contacts results in an ensemble of conformations with different relative orientations of these two subdomains.

\section{Stabilization by DNA binding}

In its native state, CylR2 binds with nanomolar affinity to the inverted repeat sequence 1 in the cytolysin promoter region ${ }^{36}$. To obtain insight into the impact of DNA binding on the stability of CylR2, we exposed CylR2 to decreasing temperatures in the presence of a 22-base-pair DNA fragment of the inverted repeat sequence. When bound to DNA, temperature-induced chemical shift changes were linear (Supplementary Fig. 15). In addition, the hydrodynamic radius of the complex remained at its native state value, demonstrating that the complex was stable in supercooled solution. Thus, the helix-turn-helix motif and the dimer arrangement were stabilized by DNA binding.

\section{DISCUSSION}

Denaturation by low temperature is a basic property of proteins that can provide insight into protein folding. Cold-denatured states can fold back to their native state on a submillisecond timescale ${ }^{33}$. In addition, a tight correlation between the impact of chemical denaturants on the ensemble of conformers at $25^{\circ} \mathrm{C}$ and the structural features that remain at low temperatures supports the relevance of cold-denatured states for the study of protein folding ${ }^{39}$.

Here we have determined seven three-dimensional structures of the E. faecalis repressor protein CylR2 at temperatures from $25^{\circ} \mathrm{C}$ to $-16^{\circ} \mathrm{C}$ (Fig. 4). The seven three-dimensional structures revealed a destabilization of the dimer interface at $-3{ }^{\circ} \mathrm{C}$, pointing to the onset of unfolding. Further decrease of the temperature to $-7^{\circ} \mathrm{C}$ resulted in the population of both a dimeric and a monomeric species. The three-dimensional structure of the monomeric intermediate of CylR2 and the subunit structure in the native-state dimer were highly similar, with the exception of the antiparallel terminal $\beta$-strand that was destabilized in the monomeric intermediate. In contrast, at $-11{ }^{\circ} \mathrm{C}$, non-native side chain interactions within a native-like monomer were observed. The non-native contacts involved side chains that form the dimer interface in the native state, suggesting that formation of non-native contacts was connected to dimer dissociation. At even lower temperatures, the packing of many internal side chains in the monomeric intermediate was perturbed, and longrange interactions to the helix-turn-helix motif were lost. However, native-like methyl-methyl contacts remained in the core formed by helices $\alpha 1, \alpha 4$ and $3_{10}$. This persistent core forms the dimer interface in the native state of CylR2 (Fig. 6 and Supplementary Fig. 16). As proteins of the helix-turn-helix family bind DNA in a dimeric form, the structure of the monomeric intermediate establishes a tight link between folding and function of homodimeric proteins. 
Previous studies have suggested that protein folding involves the successive addition of subunit structures with native-like packing ${ }^{3,18,40}$. The sequence of three-dimensional structures observed for CylR2 as temperatures decrease is in line with this mechanism. In particular, the foldon formed by the helix-turn-helix motif folds onto the folding core that comprises helices $\alpha 1, \alpha 4$ and $3_{10}$ (Fig. 6). Moreover, the experimentally observed methyl-methyl contacts (Fig. 6a and Supplementary Figs. 5 and 9) demonstrate that both well-folded and dynamic regions exist within partially folded intermediates.

The three-dimensional structures of the intermediates of CylR2 that were populated during cold-induced unfolding reveal detailed insight into the unfolding of secondary structure motifs. In helical regions, the ${ }^{1} \mathrm{H}-{ }^{15} \mathrm{~N}$ chemical shifts of most of the amide protons changed linearly with decreasing temperature, including those in the helix-turn-helix motif. The linear temperature dependence of chemical shifts together with the presence of helix-like carbon chemical shifts (Supplementary Fig. 13) indicate that hydrogen bonds in helical regions remain present down to $-16^{\circ} \mathrm{C}$. In addition, these data show that the helix-turn-helix motif was not fully unfolded despite the loss of long-range order (Supplementary Fig. 14). The NMR experiments were furthermore consistent with $\mathrm{CD}$ measurements in which no detectable loss of helical signal occurs down to $-8{ }^{\circ} \mathrm{C}$ (Supplementary Fig. 1b).

The stability of the helix-turn-helix motif as temperatures decrease is also in agreement with previous observations that the helix-turn-helix motif can fold independently ${ }^{41}$. At the same time, its stability is clearly distinct from the temperature sensitivity of the intramolecular $\beta$-sheet in CylR2. Already at $-11{ }^{\circ} \mathrm{C}$, backbone dynamics were strongly increased (Fig. 2c) and the terminal $\beta$-sheet was lost (Fig. 4 and Supplementary Fig. 7). Even in this destabilized state, however, native-like contacts of the side chains of Ile3, Gln62 and Gln64 remained (Supplementary Fig. 8). During protein folding, $\beta$-sheets might therefore form by first establishing key contacts between side chains followed by formation of interstrand hydrogen bonds. In contrast, supersecondary structures formed by $\alpha$-helices might appear early during protein folding and be subsequently stabilized by long-range interactions.

A large number of NOE distance restraints resulted in a welldefined three-dimensional structure of the monomeric intermediate of CylR2 at $-11{ }^{\circ} \mathrm{C}$ (Supplementary Table 3). At the same time, the ${ }^{15} \mathrm{~N}-{ }^{1} \mathrm{H}$ NOE was reduced to an average value of 0.27 (Fig. 2c). The considerable decrease in ${ }^{15} \mathrm{~N}-{ }^{1} \mathrm{H}$ NOE demonstrates that ps-to-ns dynamics of the backbone were strongly enhanced in the monomeric intermediate despite an extensive number of nativelike tertiary interactions. The strong increase in dynamics precludes an analysis of the backbone dynamics by the model-free approach ${ }^{42}$. However, an increase in fast internal motions from 20 ps to 45 ps, together with a decrease in the Lipari-Szabo order parameter from 0.85 to 0.7 , could account for the observed decrease in the ${ }^{15} \mathrm{~N}-{ }^{1} \mathrm{H}$ NOE (Supplementary Table 1). The ${ }^{15} \mathrm{~N}-{ }^{1} \mathrm{H}$ NOE observed for the native-like monomeric intermediate of CylR2 is comparable to that seen in parts of the molten globule of $\alpha$-lactalbumin ${ }^{43}$ but is more pronounced than that for a folding intermediate of a mutant of the IM7 protein ${ }^{44}$. In contrast, the folding intermediates of mutated engrailed homodomain and redesigned T4 lysozyme were shown to have rigid backbones comparable to those of the native state $e^{13,14}$. The presence of rigid backbones of mutated engrailed homodomain and redesigned T4 lysozyme might not only be inherent to the approach of protein engineering that destabilizes the native state and stabilizes the intermediate but also highlights the range of structural and dynamical properties present in protein folding intermediates.

The monomeric unfolding intermediates of CylR2 retained a native-like backbone topology (Fig. 4), consistent with previous studies that observed native-like backbone topologies in folding intermediates of $\alpha$-lactalbumin, redesigned T4 lysozyme and apo cytochrome $b_{562}$ (refs. 12,13,18). At the same time, experimental and theoretical studies suggest that both native and non-native interactions occur during protein folding ${ }^{1,11,12,14,16,23,24}$. During coldinduced dissociation and unfolding of CylR2, NOE contacts directly prove the presence of non-native interactions at $-11^{\circ} \mathrm{C}$ (Fig. 5 and Supplementary Fig. 11). The hydrophobic side chains of Leu43 and Leu57, which are located in the native dimer interface, rotate upon dissociation of the dimer and were buried by non-native contacts in the core of the monomeric intermediate (Fig. 5). Thus, formation of non-native contacts in the intermediate is linked to dimerization in the native state. As dimer formation is essential for DNA binding of CylR2, our results support the hypothesis that functional constraints have an important role in the formation of folding intermediates and determine their structural features ${ }^{16}$.

Protein intermediates have a key role not only in the folding of proteins but also in their pathogenic misfolding. In addition, dissociation of partially unfolded monomeric states from an oligomeric state can be rate limiting for protein aggregation ${ }^{45-47}$. A key example is the homotetrameric protein transthyretin, where tetramer dissociation and partial unfolding of the monomer precedes amyloid formation $^{46,47}$. The seven three-dimensional structures of CylR2 (Fig. 4) suggest a reason why dissociation and unfolding of a monomeric intermediate might be required for efficient aggregation. At $-11{ }^{\circ} \mathrm{C}$, the CylR2 monomer is well defined, as reflected by a large number of NOE distance restraints (Supplementary Table 3). A unique feature of this monomeric intermediate is that non-native contacts maximize hydrophobic burial (Fig. 5). Only when the temperature was further reduced were long-range interactions lost (Figs. 4 and 6) and did hydrophobic side chains become accessible for self-association and aggregation. Thus, non-native interactions seem to be important for stabilization of the monomeric intermediate and inhibition of aggregation. The observation that non-native interactions stabilize the monomeric intermediate of CylR2 is in contrast to observations made for a folding intermediate of the Fyn $\mathrm{SH} 3$ domain, where non-native interactions were suggested to lead to transient exposure of aggregation-prone regions ${ }^{6}$.

In summary, we have demonstrated that NMR spectroscopy allows the characterization of the unfolding of the homodimeric CylR2 protein at atomic resolution. The derived conformational ensembles of unfolding intermediates provide unique insights into the mechanisms of folding and unfolding of secondary, tertiary and quaternary structure in proteins.

Received 19 September 2012; accepted 15 January 2013; published online 10 February 2013

\section{METHODS}

Methods and any associated references are available in the online version of the paper.

Accession codes. Protein Data Bank (PDB): the structural ensembles of CylR2 together with NOE and torsion angle restraints at different temperatures have been deposited with accession codes 2LYJ, 2LYK, 2LYL, 2LYP, 2LYQ, 2LYR and 2LYS. Biological Magnetic Resonance Bank (BMRB): chemical shifts assigned at different temperatures have been deposited with accession codes 17892, 17893, 17894, 17895, 17896, 17897 and 17898.

\section{References}

1. Brockwell, D.J. \& Radford, S.E. Intermediates: ubiquitous species on folding energy landscapes? Curr. Opin. Struct. Biol. 17, 30-37 (2007).

2. Fersht, A.R. From the first protein structures to our current knowledge of protein folding: delights and scepticisms. Nat. Rev. Mol. Cell Biol. 9, 650-654 (2008).

3. Englander, S.W., Mayne, L. \& Krishna, M.M. Protein folding and misfolding: mechanism and principles. Q. Rev. Biophys. 40, 287-326 (2007). 
4. Canet, D. et al. Local cooperativity in the unfolding of an amyloidogenic variant of human lysozyme. Nat. Struct. Biol. 9, 308-315 (2002).

5. Khurana, R. et al. Partially folded intermediates as critical precursors of light chain amyloid fibrils and amorphous aggregates. Biochemistry 40, 3525-3535 (2001).

6. Neudecker, P. et al. Structure of an intermediate state in protein folding and aggregation. Science 336, 362-366 (2012).

7. Kiefhaber, T. Protein folding kinetics. Methods Mol. Biol. 40, 313-341 (1995)

8. Bernstein, R., Schmidt, K.L., Harbury, P.B. \& Marqusee, S. Structural and kinetic mapping of side-chain exposure onto the protein energy landscape. Proc. Natl. Acad. Sci. USA 108, 10532-10537 (2011).

9. Fersht, A. \& Winter, G. Protein engineering. Trends Biochem. Sci. 17, 292-295 (1992).

10. Balbach, J. et al. Protein folding monitored at individual residues during a two-dimensional NMR experiment. Science 274, 1161-1163 (1996).

11. Feng, H., Takei, J., Lipsitz, R., Tjandra, N. \& Bai, Y. Specific non-native hydrophobic interactions in a hidden folding intermediate: implications for protein folding. Biochemistry 42, 12461-12465 (2003).

12. Feng, H., Zhou, Z. \& Bai, Y. A protein folding pathway with multiple folding intermediates at atomic resolution. Proc. Natl. Acad. Sci. USA 102, 5026-5031 (2005).

13. Kato, H., Feng, H. \& Bai, Y. The folding pathway of T4 lysozyme: the high-resolution structure and folding of a hidden intermediate. J. Mol. Biol. $365,870-880$ (2007).

14. Religa, T.L., Markson, J.S., Mayor, U., Freund, S.M. \& Fersht, A.R. Solution structure of a protein denatured state and folding intermediate. Nature 437, 1053-1056 (2005).

15. Zhou, Z., Feng, H., Ghirlando, R. \& Bai, Y. The high-resolution NMR structure of the early folding intermediate of the Thermus thermophilus ribonuclease H. J. Mol. Biol. 384, 531-539 (2008).

16. Friel, C.T., Smith, D.A., Vendruscolo, M., Gsponer, J. \& Radford, S.E. The mechanism of folding of $\operatorname{Im} 7$ reveals competition between functional and kinetic evolutionary constraints. Nat. Struct. Mol. Biol. 16, 318-324 (2009).

17. Redfield, C. NMR studies of partially folded molten-globule states. Methods Mol. Biol. 278, 233-254 (2004).

18. Schulman, B.A., Kim, P.S., Dobson, C.M. \& Redfield, C. A residue-specific NMR view of the non-cooperative unfolding of a molten globule. Nat. Struct. Biol. 4, 630-634 (1997).

19. Meinhold, D.W. \& Wright, P.E. Measurement of protein unfolding/refolding kinetics and structural characterization of hidden intermediates by NMR relaxation dispersion. Proc. Natl. Acad. Sci. USA 108, 9078-9083 (2011).

20. Neudecker, P., Lundstrom, P. \& Kay, L.E. Relaxation dispersion NMR spectroscopy as a tool for detailed studies of protein folding. Biophys. J. 96, 2045-2054 (2009).

21. Palmer, A.G. III. NMR characterization of the dynamics of biomacromolecules. Chem. Rev. 104, 3623-3640 (2004).

22. Vallurupalli, P., Hansen, D.F., Stollar, E., Meirovitch, E. \& Kay, L.E. Measurement of bond vector orientations in invisible excited states of proteins. Proc. Natl. Acad. Sci. USA 104, 18473-18477 (2007).

23. Korzhnev, D.M., Religa, T.L., Banachewicz, W., Fersht, A.R. \& Kay, L.E. A transient and low-populated protein-folding intermediate at atomic resolution. Science 329, 1312-1316 (2010).

24. Korzhnev, D.M. et al. Low-populated folding intermediates of Fyn SH3 characterized by relaxation dispersion NMR. Nature 430, 586-590 (2004).

25. Neudecker, P., Zarrine-Afsar, A., Davidson, A.R. \& Kay, L.E. $\Phi$-value analysis of a three-state protein folding pathway by NMR relaxation dispersion spectroscopy. Proc. Natl. Acad. Sci. USA 104, 15717-15722 (2007).

26. Bouvignies, G. et al. Solution structure of a minor and transiently formed state of a T4 lysozyme mutant. Nature 477, 111-114 (2011).

27. Lindorff-Larsen, K., Piana, S., Dror, R.O. \& Shaw, D.E. How fast-folding proteins fold. Science 334, 517-520 (2011).

28. Piana, S., Lindorff-Larsen, K. \& Shaw, D.E. How robust are protein folding simulations with respect to force field parameterization? Biophys. J. 100, L47-L49 (2011).

29. Babu, C.R., Hilser, V.J. \& Wand, A.J. Direct access to the cooperative substructure of proteins and the protein ensemble via cold denaturation. Nat. Struct. Mol. Biol. 11, 352-357 (2004).

30. Privalov, P.L. Cold denaturation of proteins. Crit. Rev. Biochem. Mol. Biol. 25, 281-305 (1990)

31. Szyperski, T. \& Mills, J.L. NMR-based structural biology of proteins in supercooled water. J. Struct. Funct. Genomics 12, 1-7 (2011)

32. Foguel, D. \& Silva, J.L. Cold denaturation of a repressor-operator complex: the role of entropy in protein-DNA recognition. Proc. Natl. Acad. Sci. USA 91, 8244-8247 (1994).
33. Wong, K.B., Freund, S.M. \& Fersht, A.R. Cold denaturation of barstar: ${ }^{1} \mathrm{H},{ }^{15} \mathrm{~N}$ and ${ }^{13} \mathrm{C}$ NMR assignment and characterisation of residual structure. J. Mol. Biol. 259, 805-818 (1996).

34. Davidovic, M., Mattea, C., Qvist, J. \& Halle, B. Protein cold denaturation as seen from the solvent. J. Am. Chem. Soc. 131, 1025-1036 (2009).

35. Lassalle, M.W., Li, H., Yamada, H., Akasaka, K. \& Redfield, C. Pressureinduced unfolding of the molten globule of all-Ala $\alpha$-lactalbumin. Protein Sci. 12, 66-72 (2003)

36. Haas, W., Shepard, B.D. \& Gilmore, M.S. Two-component regulator of Enterococcus faecalis cytolysin responds to quorum-sensing autoinduction. Nature 415, 84-87 (2002).

37. Skalicky, J.J., Sukumaran, D.K., Mills, J.L. \& Szyperski, T. Toward structural biology in supercooled water. J. Am. Chem. Soc. 122, 3230-3231 (2000).

38. Kosen, P.A. Spin labeling of proteins. Methods Enzymol. 177, 86-121 (1989).

39. Campbell, J.C. \& Whitten, S.T. Mutational analysis of m-values as a strategy to identify cold-resistant substructures of the protein ensemble. Proteins $\mathbf{8 0}$ 184-193 (2012).

40. Vendruscolo, M., Paci, E., Dobson, C.M. \& Karplus, M. Three key residues form a critical contact network in a protein folding transition state. Nature 409, 641-645 (2001).

41. Religa, T.L. et al. The helix-turn-helix motif as an ultrafast independently folding domain: the pathway of folding of Engrailed homeodomain. Proc. Natl. Acad. Sci. USA 104, 9272-9277 (2007).

42. Lipari, G. \& Szabo, A. Model-free approach to the interpretation of nuclear magnetic-resonance relaxation in macromolecules. 1. Theory and range of validity. J. Am. Chem. Soc. 104, 4546-4559 (1982).

43. Ramboarina, S. \& Redfield, C. Probing the effect of temperature on the backbone dynamics of the human $\alpha$-lactalbumin molten globule. J. Am. Chem. Soc. 130, 15318-15326 (2008).

44. Whittaker, S.B., Spence, G.R., Gunter Grossmann, J., Radford, S.E. \& Moore, G.R. NMR analysis of the conformational properties of the trapped on-pathway folding intermediate of the bacterial immunity protein $\operatorname{Im} 7$. J. Mol. Biol. 366, 1001-1015 (2007).

45. Ecroyd, H., Thorn, D.C., Liu, Y. \& Carver, J.A. The dissociated form of $\kappa$-casein is the precursor to its amyloid fibril formation. Biochem. J. 429, 251-260 (2010).

46. Quintas, A., Saraiva, M.J. \& Brito, R.M. The tetrameric protein transthyretin dissociates to a non-native monomer in solution. A novel model for amyloidogenesis. J. Biol. Chem. 274, 32943-32949 (1999).

47. Quintas, A., Vaz, D.C., Cardoso, I., Saraiva, M.J. \& Brito, R.M. Tetramer dissociation and monomer partial unfolding precedes protofibril formation in amyloidogenic transthyretin variants. J. Biol. Chem. 276, 27207-27213 (2001).

\section{Acknowledgments}

We thank N. Rezaei-Ghaleh for useful discussions. This work was supported by the START and Ventures Programmes of Foundation for Polish Science (Fundacja na rzecz Nauki Polskiej; http://www.fnp.org.pl/) operated within the Innovative Economy Operational Programme (IE OP) 2007-2013 within European Regional Development Fund (L.J. and M.J.), the Iuventus Plus project no. IP2011 019471 from Polish Ministry of Sciences and Higher Education (M.J.) and the Cluster of Excellence and Deutsche Forschungsgemeinschaft Research Center "Nanoscale Microscopy and Molecular Physiology of the Brain" (to M.Z.). C.D.S. was supported by the Intramural Research Program of the Center for Information Technology at the US National Institutes of Health

\section{Author contributions}

M.J. performed structure determination, data acquisition and data analysis, and contributed to writing the paper. L.J. performed structure determination, data acquisition and data analysis, and contributed to writing the paper. H.-Y.K. performed data acquisition and analysis. M.-K.C. analyzed data, C.D.S. performed ensemble calculations, K.G. and S.B. prepared samples, and M.Z. performed data acquisition, designed and supervised the project and wrote the paper.

\section{Competing financial interests}

The authors declare no competing financial interests.

\section{Additional information}

Supplementary information is available in the online version of the paper. Reprints and permissions information is available online at http://www.nature.com/reprints/index. html. Correspondence and requests for materials should be addressed to M.Z. 


\section{ONLINE METHODS}

Sample preparation. Mutants of CylR2 (T55C, N40C, L6S and I49S) were generated by using the QuikChange site-directed mutagenesis kit (Stratagene). The introduced mutations were confirmed by DNA sequencing. Details of cloning, protein overexpression and purification have been described elsewhere ${ }^{48}$. ${ }^{13} \mathrm{C}$ - and ${ }^{15} \mathrm{~N}$-labeled samples were prepared from Escherichia coli cells grown in M9-based minimal medium containing ${ }^{15} \mathrm{NH}_{4} \mathrm{Cl}$ and $\left[{ }^{13} \mathrm{C}\right]$ glucose. A sample of total volume of $540 \mu \mathrm{L}$ in a regular NMR tube containing $0.55 \mathrm{mM}$ of protein (monomer subunit concentration) in $93 \% / 7 \% \mathrm{H}_{2} \mathrm{O} / \mathrm{D}_{2} \mathrm{O}, 50 \mathrm{mM}$ HEPES buffer and $600 \mathrm{mM} \mathrm{NaCl}$ at $\mathrm{pH} 7.0$ was used for the NMR measurements. For measurements below $-7{ }^{\circ} \mathrm{C}$, the protein was transferred into ten glass capillaries of $1.0-\mathrm{mm}$ outer diameter, giving a total volume of the sample of approximately $260 \mu \mathrm{L}$. The ten capillaries were placed into a 5-mm NMR tube for NMR measurements.

MTSL ((1-oxy-2,2,5,5-tetramethyl-D-pyrroline-3-methyl)-methanethiosulfonate, Toronto Research Chemicals) labeling was performed as recently described ${ }^{49}$. Excess MTSL was removed by reverse phase HPLC ${ }^{50}$. ESI-MS was used to verify that all unbound MTSL had been removed.

The 22-bp oligonucleotide (5'-TTGTCAAGTGACACTTGACAAA- ${ }^{\prime}$ ) used for NMR measurements was purchased from Eurogentec (Liège, Belgium). The oligonucleotide was resuspended in $5 \mathrm{mM}$ HEPES, pH 7.6, $0.05 \mathrm{M} \mathrm{NaCl}$ and $0.01 \mathrm{M} \mathrm{MgCl}_{2}$ and annealed by heating to $100{ }^{\circ} \mathrm{C}$ for $5 \mathrm{~min}$ in a heating block and cooling down slowly to room temperature. The annealed DNA was dialyzed against water, lyophilized and dissolved in the same buffer as the protein. The NMR sample of the CylR2-DNA complex contained $100 \mu \mathrm{M}$ ${ }^{15} \mathrm{~N}$-labeled CylR2 and $100 \mu \mathrm{M}$ DNA.

NMR spectroscopy. NMR spectra were recorded on a Bruker $700 \mathrm{MHz}$ Avance III spectrometer equipped with a z-axis gradient TXI probe (Bruker Biospin, Germany). Temperatures were adjusted on the basis of a reference sample containing methanol. On the basis of previously published assignments of CylR2 in $350 \mathrm{mM} \mathrm{NaCl}, 25^{\circ} \mathrm{C}$ (BMRB code 6317), sequence-specific assignments at different temperatures were obtained using two-dimensional ${ }^{1} \mathrm{H}-{ }^{15} \mathrm{~N}$ HSQC, ${ }^{1} \mathrm{H}-{ }^{13} \mathrm{C}$ HSQC, three-dimensional CCH-TOCSY, three-dimensional ${ }^{15} \mathrm{~N}$-edited NOESY-HSQC and three-dimensional ${ }^{13} \mathrm{C}$-edited NOESY-HSQC NMR experiments. Three-dimensional ${ }^{13} \mathrm{C}$-edited NOESY-HSQC spectra were recorded with the offset, spectral widths and ${ }^{13} \mathrm{C}-{ }^{-1} \mathrm{H}$ coupling constants tuned to aliphatic and aromatic carbons (Supplementary Table 2). Backbone assignments of the single-point mutants (L6S, I49S) were derived from threedimensional HNCA, three-dimensional $\mathrm{CBCA}(\mathrm{CO}) \mathrm{NH}$ and three-dimensional HNCACB experiments (Supplementary Table 2).

For determination of hydrodynamic radii, CylR2 was dissolved in $90 \% \mathrm{H}_{2} \mathrm{O}$ / $10 \% \mathrm{D}_{2} \mathrm{O}, 50 \mathrm{mM}$ HEPES, $\mathrm{pH} 7.0$, with $\sim 1 \%$ of dioxane as an internal radius standard and viscosity probe. A series of one-dimensional ${ }^{1} \mathrm{H}$ spectra were collected using the Bruker pulse program pgsled. The gradient strength was linearly increased from $5 \%$ to $95 \%$ of the maximum value in 16 steps, with $100 \%$ gradient strength corresponding to $56.9 \mathrm{G} / \mathrm{cm}$. For each ${ }^{1} \mathrm{H}$ spectrum, 128 scans and 16,000 complex data points with a spectral width of $6,000 \mathrm{~Hz}$ were acquired. Using Topspin 2.0 (Bruker Biospin, Germany), the signals corresponding to the aliphatic region of the ${ }^{1} \mathrm{H}$ spectra (3.3-0.5 p.p.m.) were integrated. To determine the apparent diffusion coefficient of the target protein ( $\left.\mathrm{d}^{\text {prot}}\right)$, signal decays were fitted as a function of gradient strength to Gaussian functions. The same procedure was applied for the dioxane peak, and the diffusion coefficient of dioxane ( $\left.\mathrm{d}^{\text {ref }}\right)$ was measured. Stokes radii of CylR2 were calculated from the apparent diffusion coefficients of CylR2 and dioxane using its known Stokes radius $(2.12 \AA)$. Errors were estimated by duplicated measurements.

The global correlation time $\tau_{c}$ of CylR2 at different temperatures was estimated from experimental amide proton $T_{2}$ relaxation times according to the relation: $\tau_{\mathrm{c}}$ (ns) $\sim 1 /\left(5 T_{2}(\mathrm{~s})\right.$ ). Proton $T_{2}$ relaxation times at selected temperatures were measured using 1-1 echo experiments ${ }^{51}$ with delays $(t)$ of $0.2 \mathrm{~ms}$, $1.2 \mathrm{~ms}, 3.1 \mathrm{~ms}, 4.5 \mathrm{~ms}, 6.0 \mathrm{~ms}, 8.0 \mathrm{~ms}$ and $12.2 \mathrm{~ms}$. The average amide proton $T_{2}$ relaxation time was then determined using a simple exponential fit of the resulting intensities for each delay $t$ to the equation $I_{\mathrm{t}}=I_{0} \exp \left(-2 t / T_{2}\right)$. Errors in $\tau_{\mathrm{c}}$ were estimated from three repeat measurements.

Line widths of amide protons were extracted from two-dimensional ${ }^{1} \mathrm{H}-{ }^{15} \mathrm{~N}$ HSQC spectra that were processed without a window function. Steady-state heteronuclear ${ }^{15} \mathrm{~N}-{ }^{1} \mathrm{H}$ NOE values were calculated on the basis of intensity ratios of the ${ }^{15} \mathrm{~N}-{ }^{1} \mathrm{H}$ correlation peaks from pairs of interleaved spectra acquired with and without ${ }^{1} \mathrm{H}$ presaturation during the recycle time of $7 \mathrm{~s}$. Errors were derived on the basis of $S / N$ values for ${ }^{15} \mathrm{~N}-{ }^{1} \mathrm{H}$ correlation peak heights, with $\left(I_{\mathrm{NOE} i}\right)$ and without $\left(I_{\text {noNOEi }}\right)^{1} \mathrm{H}$ presaturation obtained in Sparky according to the following equation:

$$
\sigma_{\mathrm{NOE} i}=\left|\mathrm{NOE}_{i}\right|\left[1 /\left(S / N_{\mathrm{NOE} i}\right)^{2}+1 /\left(S / N_{n o \mathrm{NOE} i}\right)^{2}\right]^{1 / 2}
$$

where $\mathrm{NOE}_{i}=I_{\mathrm{NOE} i} / I_{\mathrm{noNOE} i}$. PRE intensity ratios were obtained from intensities of cross-peaks of backbone amide proton-nitrogen pairs in two-dimensional ${ }^{1} \mathrm{H}-{ }^{15} \mathrm{~N}$ HSQC spectra of the paramagnetic $\left(I_{\text {para }}\right)$ and diamagnetic $\left(I_{\text {dia }}\right)$ state. The diamagnetic reference state was obtained by addition of a two- to threemolar excess of ascorbic acid to the MTSL-loaded sample. Theoretical intensity ratios $I_{\text {para }} / I_{\mathrm{dia}}$ were back-calculated from each conformer of the ensembles at different temperatures according to the equation in ref. 52

$$
\frac{I_{\text {para }}}{I_{\text {dia }}}=\frac{R_{2} \exp \left(-R_{2}^{\text {para }} t\right)}{R_{2}+R_{2}^{\text {para }}}
$$

in which $R_{2}^{\text {para }}$ is the enhancement of the transverse relaxation rate by the unpaired electron, $t$ is the total INEPT evolution time of the two-dimensional ${ }^{1} \mathrm{H}-{ }^{15} \mathrm{~N}$ HSQC ( $\left.~ 9.0 \mathrm{~ms}\right)$ and $R_{2}$ values are the intrinsic transverse relaxation times of the amide protons estimated from 1-1 echo experiments (described above). Assuming a rigid spin label ${ }^{53}$, the enhancement of the transverse relaxation rate by the unpaired electron $\left(R_{2}^{\text {para }}\right)$ was calculated according to

$$
R_{2}^{\text {para }}=\frac{K}{r^{6}}\left(4 \tau_{c}+\frac{3 \tau_{c}}{1+\omega_{h}^{2} \tau_{c}^{2}}\right)
$$

in which $r$ is the distance between the unpaired electron (approximated by the position of the $\mathrm{C}_{\beta}$ atom of the side chain to which MTSL was attached) and the amide proton, $K$ is $1.23 \times 10^{-32} \mathrm{~cm}^{6} \mathrm{~s}^{-2}$, and $\omega_{h}$ is the Larmor frequency of the proton. $\tau_{c}$ is the correlation time for the electron-nuclear interaction and was set to the global correlation time of CylR2, which was estimated from amide proton $T_{2}$ relaxation times (described above). Variations of up to $20 \%$ in the estimated values of $\tau_{c}$ and $R_{2}$ only weakly affect the theoretical PRE profiles. Ensemble-averaged PREs were obtained by calculating the linear average of predicted intensity ratios for each of the 20 conformers in the ensemble shown in Figure 4.

Estimation of dimer fractions. The percentage of dimeric molecules was estimated from the temperature dependence of the hydrodynamic radius (Fig. 3a). The reaction constant $\left(K_{\mathrm{d}}\right)$ is related to the free energy $(\Delta G)$ of the reaction process according to $\Delta G=\Delta H-T \Delta S=-\mathrm{RT} \ln \left(K_{\mathrm{d}}\right)$. When the reaction rate of the process of protein dimer dissociation is known, the activation energy $E^{\text {a }}(\sim \Delta H)$ can be calculated using the well-known Van't Hoff-type model, which describes the relation between the reaction rate $K_{\mathrm{d}}$ and temperature $T^{54,55}$. If we assume that $\Delta H$ and $\Delta S$ are independent of temperature over the investigated range $\left(-10{ }^{\circ} \mathrm{C}\right.$ to $\left.-3{ }^{\circ} \mathrm{C}\right)$, we can use the fitting results to calculate $\Delta H$ and $\Delta S$. A linear fit to the experimental values of the hydrodynamic radii is in agreement with the Van't Hoff-type relationship $\left(R^{2}=0.991\right)$. Calculated activation energy $\left(E^{\mathrm{a}}\right)$ and entropy $(\Delta S)$ of the dimer dissociation reaction were $-725.09 \pm 48.4 \mathrm{~kJ} / \mathrm{mol}$ and $-2,784.1 \pm 182 \mathrm{~J} /(\mathrm{mol} \cdot \mathrm{K})$, respectively.

On the basis of the experimental values of the hydrodynamic radius, the molar ratio of the dimer and monomer concentration $([D] /[M])$ were subsequently calculated for each temperature using the relation between the hydrodynamic radius and the concentration of the monomeric $[M]$ and dimeric $[D]$ species according to $\left\langle R_{\mathrm{h}}>_{\mathrm{T}}=([M]+4[D]) /\left([M] / R_{\mathrm{hm}}+4[D] / R_{\mathrm{hd}}\right)\right.$, where $\left\langle R_{\mathrm{h}}>_{\mathrm{T}}\right.$ is the hydrodynamic radius experimentally determined at a certain temperature ${ }^{54}$. $R_{\mathrm{hm}}$ and $R_{\mathrm{hd}}$ are the hydrodynamic radii of the monomer at $-10^{\circ} \mathrm{C}(15.5 \AA)$ and the dimer at $25^{\circ} \mathrm{C}(22.9 \AA)$, respectively; $K_{\mathrm{d}}=[M]^{2} /[D]$ with $[M]=0.25\left(-K_{\mathrm{d}}+\left(K_{\mathrm{d}}^{2}+8 \mathrm{~K}_{\mathrm{d}}[C]\right)^{0.5}\right)$, with $[C]=[M]+2[D]$. $[M]$ was $0.5 \mathrm{mM}$ and $[D]=0.125\left(K_{\mathrm{d}}+4[C]-\left(K_{\mathrm{d}}^{2}+8 K_{\mathrm{d}}[C]\right)^{0.5}\right)$.

In addition, the percentage of dimeric molecules was estimated from the intensity of the well-separated cross-peak of $\mathrm{H} \delta 2 / \mathrm{H} \delta 3 / \mathrm{C} \delta$ from Lys 48 (Fig. 4a), which is involved in intermolecular $\mathrm{C}-\mathrm{H}-\pi$ interaction with the aromatic moiety of Trp63. The two-dimensional ${ }^{1} \mathrm{H}-{ }^{13} \mathrm{C}$ HSQC aliphatic spectra measured at temperatures of $25^{\circ} \mathrm{C},-3{ }^{\circ} \mathrm{C}$ and $-7{ }^{\circ} \mathrm{C}$ were used. Cross-peak intensities at $25^{\circ} \mathrm{C}$ were assumed to represent $100 \%$ of dimer, and the peak intensity of the well-separated $\mathrm{H} \beta / \mathrm{C} \beta$ cross peak of Ala51 was used for internal scaling of peak intensities between different temperatures. The fraction of dimer species 
remaining at a certain temperature $T$ below $25^{\circ} \mathrm{C}$ was estimated from the ratio of Lys 48 side chain cross-peak intensities $\left(x_{\mathrm{dimer}, \mathrm{T}}=I_{\mathrm{H} \delta 2 / \mathrm{H} \delta 3, \mathrm{~T}} / I_{\mathrm{H} \delta 2 / \mathrm{H} 83,25^{\circ} \mathrm{C}}\right)$ and was in agreement with results from hydrodynamic radius measurements.

Structure calculations. At each temperature, the initial structure calculations were done with the program CYANA 3.0 (ref. 56). Distance restraints were derived from aliphatic versions of three-dimensional ${ }^{13} \mathrm{C}$-edited NOESY-HSQC spectra at $25^{\circ} \mathrm{C},-3{ }^{\circ} \mathrm{C},-7{ }^{\circ} \mathrm{C},-11^{\circ} \mathrm{C},-14{ }^{\circ} \mathrm{C}$ and $-16^{\circ} \mathrm{C}$; aromatic versions of three-dimensional ${ }^{13} \mathrm{C}$-edited NOESY-HSQC experiments at $25^{\circ} \mathrm{C},-3{ }^{\circ} \mathrm{C}$, $-7{ }^{\circ} \mathrm{C}$ and $-14{ }^{\circ} \mathrm{C}$; and three-dimensional ${ }^{15} \mathrm{~N}$-edited NOESY-HSQC spectra at $25^{\circ} \mathrm{C},-3^{\circ} \mathrm{C},-7^{\circ} \mathrm{C}$ and $-11^{\circ} \mathrm{C}$ (Supplementary Table 2). Owing to severe line broadening, only a very small number of cross-peaks was observed in a three-dimensional ${ }^{15} \mathrm{~N}$-edited NOESY-HSQC spectrum at $-11{ }^{\circ} \mathrm{C}$, making this spectrum not useful for structural analysis at $-11^{\circ} \mathrm{C}$ and lower temperatures. NOESY mixing times were $150 \mathrm{~ms}$ and $200 \mathrm{~ms}$ for ${ }^{15} \mathrm{~N}$ - and ${ }^{13} \mathrm{C}$-edited NOESYHSQC experiments, respectively. Cross-peaks in NOESY spectra were initially assigned automatically using the program CYANA 3.0 (ref. 56) followed by careful manual inspection and verification. The $\phi$ and $\psi$ torsion angles restraints were defined on the basis of $\mathrm{H}^{\mathrm{N}}, \mathrm{N}, \mathrm{C}^{\alpha}, \mathrm{C}^{\beta}$ and $\mathrm{H}^{\alpha}$ chemical shifts at a certain temperature using the PREDITOR software server ${ }^{57}$. At $-7{ }^{\circ} \mathrm{C},-11^{\circ} \mathrm{C}$, $-14{ }^{\circ} \mathrm{C}$ and $-16^{\circ} \mathrm{C}$, no complete set of resonances for prediction of dihedral angles was available due to signal broadening. To account for the increased dynamics at these temperatures, the angle ranges predicted by PREDITOR were multiplied by a factor of two. At all temperatures except $-7^{\circ} \mathrm{C}$, the structures were folded starting from an extended strand. At $-7^{\circ} \mathrm{C}$, owing to coexistence of the dimeric and monomeric forms in approximately equal amounts (as derived from $R_{\mathrm{h}}$ measurements), ensemble calculations were performed. To this end, the Xplor-NIH software was modified to handle distance restraints for heterogeneous ensembles (this work is available starting in Xplor-NIH version 2.29). Fast exchange between monomer and dimer species allows observation of a single set of chemical shifts that was used for dihedral angle prediction. To account for possible local differences in dimer and monomer species, the angle ranges predicted by PREDITOR were multiplied by a factor of two. Starting structures for the ensemble calculations were the lowest-energy structures determined at $-11{ }^{\circ} \mathrm{C}$ and $-3{ }^{\circ} \mathrm{C}$ for the monomer and dimer, respectively. Weights were set to $50 \%$ for both the monomer and dimer. Three lists of NOE restraints were applied, one with NOE restraints specific to the dimer interface, and verified manually on the basis of the dimer structure from $-3{ }^{\circ} \mathrm{C}$, and two equal subunit/monomer-specific lists were applied. To preserve the $\mathrm{C} 2$ symmetry of the dimer, noncrystallographic symmetry and distance symmetry terms were applied. In addition, the backbone atoms of the monomer were restrained to lie within an r.m.s. deviation of $1.5 \AA$ of the starting coordinates. The 40 lowest-energy structures from all temperatures, obtained from the 200 that were submitted for simulated annealing, were refined in explicit solvent using Xplor-NIH ${ }^{58}$. The edge length of the water box was $18.8 \AA$. The final ensembles discussed in this article represent the 20 lowest energy conformers obtained after the water refinement step. They had no distance violations greater than $0.5 \AA$ and no dihedral angle violations greater than $5^{\circ}$. Statistics for the NMR ensembles of proteins structures at six different temperatures are presented in Supplementary Table 3.

Circular dichroism. Circular dichroism spectra of CylR2 were obtained on a Photophysics Chirascan spectrometer. A 1-mm path length quartz cuvette containing $50 \mu \mathrm{M}$ of protein in $50 \mathrm{mM}$ HEPES, $\mathrm{pH} 7.0,350 \mathrm{mM} \mathrm{NaCl}$ was used. Melting curves were determined by monitoring changes in ellipticity at a wavelength of $222 \mathrm{~nm}$. The temperature was increased from $55^{\circ} \mathrm{C}$ to $95^{\circ} \mathrm{C}$ at a rate of $80^{\circ} \mathrm{C} / \mathrm{h}$. For measurement of CD spectra below $20^{\circ} \mathrm{C}, 30 \mu \mathrm{L}$ of $0.42 \mathrm{mM}$ CylR2 were dissolved in $170 \mu \mathrm{L}$ of $20 \mathrm{mM}$ phosphate buffer with $100 \mathrm{mM} \mathrm{NaCl}$, $\mathrm{pH}$ 7.0. The CD spectra were recorded in the range of $190-260 \mathrm{~nm}$ at temperatures of $20^{\circ} \mathrm{C}, 15^{\circ} \mathrm{C}, 10^{\circ} \mathrm{C}, 5^{\circ} \mathrm{C}, 1^{\circ} \mathrm{C}, 0^{\circ} \mathrm{C},-1{ }^{\circ} \mathrm{C},-3{ }^{\circ} \mathrm{C},-5^{\circ} \mathrm{C}$ and $-8^{\circ} \mathrm{C}$.

48. Razeto, A. et al. Expression, purification, crystallization and preliminary crystallographic studies of the Enterococcus faecalis cytolysin repressor CylR2. Acta Crystallogr. D Biol. Crystallogr. 60, 746-748 (2004).

49. Rumpel, S., Becker, S. \& Zweckstetter, M. High-resolution structure determination of the CylR2 homodimer using paramagnetic relaxation enhancement and structure-based prediction of molecular alignment. J. Biomol. NMR 40, 1-13 (2008).

50. Gruene, T. et al. Integrated analysis of the conformation of a protein-linked spin label by crystallography, EPR and NMR spectroscopy. J. Biomol. NMR 49, 111-119 (2011).

51. Sklenar, V. \& Bax, A. Spin-echo water suppression for the generation of pure-phase two-dimensional NMR-spectra. J. Magn. Reson. 74, 469-479 (1987).

52. Battiste, J.L. \& Wagner, G. Utilization of site-directed spin labeling and high-resolution heteronuclear nuclear magnetic resonance for global fold determination of large proteins with limited nuclear Overhauser effect data. Biochemistry 39, 5355-5365 (2000).

53. Iwahara, J. \& Clore, G.M. Detecting transient intermediates in macromolecular binding by paramagnetic NMR. Nature 440, 1227-1230 (2006).

54. Aymard, P., Durand, D. \& Nicolai, T. The effect of temperature and ionic strength on the dimerisation of $\beta$-lactoglobulin. Int. J. Biol. Macromol. 19 213-221 (1996).

55. Bischof, J.C. \& He, X. Thermal stability of proteins. Ann. NY Acad. Sci. 1066, 12-33 (2005).

56. Güntert, P. Automated NMR structure calculation with CYANA. Methods Mol. Biol. 278, 353-378 (2004).

57. Berjanskii, M.V., Neal, S. \& Wishart, D.S. PREDITOR: a web server for predicting protein torsion angle restraints. Nucleic Acids Res. 34, W63-W69 (2006).

58. Schwieters, C.D., Kuszewski, J.J., Tjandra, N. \& Clore, G.M. The Xplor-NIH NMR molecular structure determination package. J. Magn. Reson. 160, 65-73 (2003). 